\title{
AZtec Wave - a New Way to Achieve Combined EDS and WDS Capability on SEM
}

Simon Burgess and Philippe Pinard

Oxford Instruments NanoAnalysis, High Wycombe, England, United Kingdom

The increasing capability of energy dispersive spectrometers (EDS) and software to accurately measure the distribution and composition of elements at ever higher speeds and lower detection limits [1-3], means a scanning electron microscope (SEM) equipped with EDS and a wavelength dispersive spectrometer (WDS) offers an ever more powerful tool for materials characterisation. EDS provides fast accurate results for major and many minor elements, and enables users to solve ever more complex quantitative analysis challenges in the SEM. The role for WDS becomes the measurement of minor or trace elements present at concentrations below the level for effective analysis by EDS. Therefore, the enhanced spectral resolution and peak to background are now the critical advantages of WDS. However, to be successful any tool that combines these techniques must allow both techniques to operate to their full potential. It must also be designed to be compatible with current SEM operating environments and workflows, making the power of this type of instrument fully accessible to users who may not be experts or even regular users of microanalysis techniques.

Spectrometers with a fully focussing geometry meet the performance requirements of WDS in the SEM best for two reasons. Firstly they cover an energy range large enough for accurate quantitative analysis of a comprehensive range of elements. For example, being able to use $\mathrm{K} \alpha$ lines for quantification of transition metals provides much higher accuracy than other spectrometers that can only detect these elements with low energy $\mathrm{L}$ lines. Secondly the resolution of the spectrometer is good enough to separate the most severe overlaps e.g. Mo L $\alpha$ / S K $\alpha$, Ti $\mathrm{K} \alpha / \mathrm{Ba} \mathrm{L} \alpha$ and $\mathrm{Ti} \mathrm{K} \alpha / \mathrm{V} \mathrm{K} \beta$. This enhanced resolution also means improved peak to background ratios allowing better detection limits to be achieved in shorter times or with lower beam currents than spectrometers with flat diffracting crystals and limited spectral resolution.

One of the major barriers to take full advantage of the strengths of EDS and WDS in the SEM is the skill level of typical SEM users, who are only familiar to SEM and EDS. We have therefore focussed on creating and testing practical workflows to make this new tool accessible to all users. There have been many attempts to do this in the past, which have tended to try to make WDS fit into already established EDS workflows. While these have delivered improvements in ease of use, they often compromised both EDS and WDS operation for most users. The critical difference between EDS and WDS is that for WDS all the decisions required to define an analysis are made before the data is acquired, while little complex data analysis is required after data collection. In contrast EDS requires little upfront thinking, with parameters easily changed as the data is collected, and the complexity in the workflow comes when analysing the data. In other words, the typical challenge for WDS users is how to collect data, whereas the challenge for EDS users is to generate useful and accurate results from the very large amounts of data that can be easily collected.

With the development of AZtec Wave, we are introducing new workflows for combined EDS and WDS analysis. These workflows and the software that delivers them is based on the AZtec environment, but critically do not attempt to integrate WDS into existing EDS software. It uses new workflows designed from scratch, with the optimised operation of EDS and WDS together as the main goal (Fig. 1). As WDS parameters are much more critical to define correctly before data collection, the workflow leads with the definition of WDS acquisition parameters, rather than EDS. However, as EDS is always present it can be used to constantly inform the set-up of WDS. For example, EDS spectra are used to find the correct area 
for analysis before data acquisition and to help calculate automatically the parameters needed for acquisition (e.g. acquisition time, background positions). Likewise this pre-definition of acquisition parameters can also include optimisation of EDS (e.g. process time, detector position) so data from both spectrometer types can be collected seamlessly and simultaneously. With the accuracy and sensitivity of Ultim Max EDS detectors at high count rates, a uniquely detailed analysis of major, minor and trace elements in the sample is possible without extending the acquisition time significantly. 0
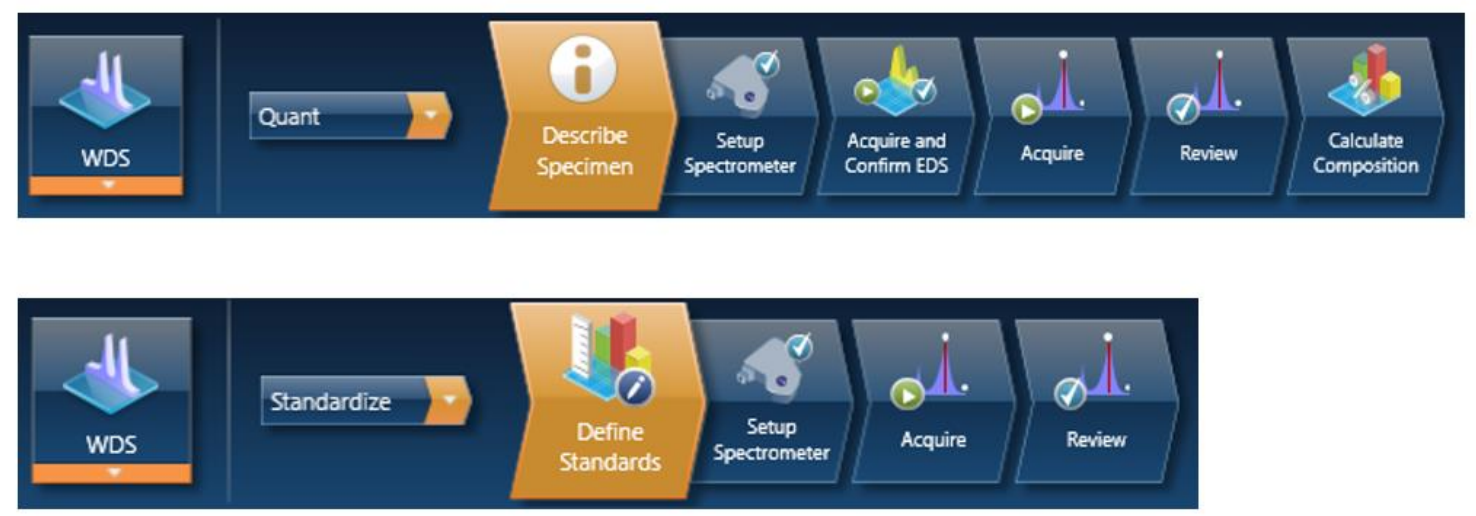

Figure 1. Figure 1. Workflows for quantification and standardization in AZtec Wave

\section{References}

[1] C Collins et al, Microsc. Microanal. 21 S3 (2015), 1627

[2] P Pinard et al, Microsc. Microanal. 24 S1 (2018), 724

[3] P Pinard et al, EMAS 2019 Proceedings (2019), 269 